\title{
Estudio Descriptivo de la Situación Nutricional en Niños de 6 - 72 Meses de la República Argentina. Resultados de la Encuesta Nacional de Nutrición y Salud (ENNyS)
}

\author{
Dr. PABLO DURÁN ${ }^{1}$, Lic. GUADALUPE MANGIALAVORI ${ }^{1}$, Lic. ANA BIGLIERI ${ }^{1}$, Bioq. LAURA KOGAN ${ }^{1}$, Dr. \\ ENRIQUE ABEYÁ GILARDON ${ }^{1}$
}

1. Dirección Nacional de Maternidad e Infancia, Ministerio de Salud de la Nación.

\section{SUMMARY \\ Nutrition status in Argentinean children 6 to 72 months old. Results from the National Nutrition and Health Survey (ENNyS)}

Introduction: Data availability on nutrition status from vulnerable population groups is essential for the design and evaluation of interventions. The Ministry of Health from Argentina Developer in 2004-2005 the National Survey of Nutrition and Health. Our objective is to describe the nutrition status of infants and preschool children (6-72 months), globally and by region and socioeconomic level. Population and Methods: A probabilistic, multi-stage sample was selected, representative at the national, regional and provincial levels. Anthropometric indices weight/age, height/age and weight/height, were estimated, according to WHO reference. Nutrient intake was estimated by a 24-hours recall, and hemoglobin, serum ferritin, retinol and vitamin D concentration were measured. Results: Prevalence of stunting, wasting and obesity were $8.0 \%, 1.3 \%$, and $10.4 \%$, respectively. Prevalence of anemia was $16.5 \%$ in children $<6$ years and $35.3 \%$ in children aged $6-23$ months. Prevalence of subclinical vitamin A deficiency in children of 2-5 years was $14.3 \%$, and prevalence of vitamin D deficiency in Patagonia was $2.8 \%$ in children aged 6-23 months. Different nutrient intake inadequacies were observed. Infants and children at the lower socioeconomic level showed higher prevalence of nutrition inadequacies. Conclusions: The coexistence of different nutrition deficiencies and overweight and obesity constitute the main characteristic of Argentinean infants and preschool children. Relevant differences exist according to socio-economic and geographic conditions.

(Key words: nutrition status, survey, malnutrition, infant, child, epidemiology).

Arch Argent Pediatr 2009; 107(5): 397-404

Correspondencia a:

Dr. Enrique Abeyá Gilardon.

E-mail: ennys@ucmisalud.ar 


\section{RESUMEN}

Introducción: La disponibilidad de información sobre el estado de salud y nutrición de la población es fundamental para planificar acciones y, en ese sentido, el Ministerio de Salud de la Nación desarrolló, en 2004-2005, la Encuesta Nacional de Nutrición y Salud (ENNyS). El objetivo del presente trabajo es describir la situación de salud y el estado nutricional en niños de 6-72 meses, según región y nivel socioeconómico. Población y Métodos: La ENNyS se realizó en una muestra probabilística, con representatividad provincial, regional y nacional según el indicador. Se estimaron los índices peso/edad, talla/edad y peso/talla, según OMS. Se valoró ingesta cuantitativamente y cualitativamente, y la distribución de hemoglobina, ferritina sérica, retinol plasmático y vitamina D, con su correspondiente prevalencia de déficit. Resultados: Se observó 8,0\% de acortamiento, $1,3 \%$ de emaciación y $10,4 \%$ de obesidad. La prevalencia de anemia fue $16,5 \%$ en menores de 6 años y $35,3 \%$ en niños de 6-23 meses. La prevalencia de deficiencia subclínica de vitamina A en niños de 2-5 años fue 14,3\% y 2,8\% de los niños de 6-23 meses en la región Patagonia presentaron déficit de vitamina D. Se observaron deficiencias alimentarias en nutrientes críticos. Los niños que pertenecen a hogares de bajo nivel socioeconómico presentaron mayor prevalencia de condiciones de deficit nutricional. Conclusiones: Se observa coexistencia de condiciones de déficit y exceso, con diferencias significativas según provincia, región o condiciones socioeconómicas.

(Palabras clave: Estado nutricional, malnutrición, niños, encuesta, epidemiología).

ESTE TRABAJO LO PUEDE ENCONTRAR EN EXTENSO EN WWW.SciELO.ORG 